\title{
On the possibility of identifying human subjects using behavioural complexity analyses
}

\author{
Petr Kloucek ${ }^{1, *}$ and Armin von Gunten ${ }^{1,2, *}$ \\ 1 CAMPsyN, SUPAA, Hôpital de Cery, Route de Cery, Lausanne University Hospital, CH-1008 Prilly, Lausanne, Switzerland \\ 2 SUPAA, Hôpital de Cery, Route de Cery, Lausanne University Hospital, CH-1008 Prilly, Lausanne, Switzerland \\ * Correspondence: petr.kloucek@chuv.ch, armin-von-gunten@chuv.ch
}

Received February 16, 2016; Revised July 13, 2016; Accepted July 13, 2016

\begin{abstract}
Background: Identification of human subjects using a geometric approach to complexity analysis of behavioural data is designed to provide a basis for a more precise diagnosis leading towards personalised medicine.

Methods: The approach is based on capturing behavioural time-series that can be characterized by a fractional dimension using non-invasive longer-time acquisitions of heart rate, perfusion, blood oxygenation, skin temperature, relative movement and steps frequency. The geometry based approach consists in the analysis of the area and centroid of convex hulls encapsulating the behavioural data represented in Euclidian index spaces based on the scaling properties of the self-similar normally distributed behavioural time-series of the above mentioned quantities.

Results: An example demonstrating the presented approach of behavioural fingerprinting is provided using sensory data of eight healthy human subjects based on approximately fifteen hours of data acquisition. Our results show that healthy subjects can be factorized to different similarity groups based on a particular choice of a convex hull in the corresponding Euclidian space. One of the results indicates that healthy subjects share only a small part of the convex hull pertaining to a highly trained individual from the geometric comparison point of view. Similarly, the presented pair-wise individual geometric similarity measure indicates large differences among the subjects suggesting the possibility of neuro-fingerprinting.

Conclusions: Recently introduced multi-channel body-attached sensors provide a possibility to acquire behavioural time-series that can be mathematically analysed to obtain various objective measures of behavioural patterns yielding behavioural diagnoses favouring personalised treatments of, e.g., neuropathologies or aging.
\end{abstract}

Keywords: behavioural complexity indexing; behavioural fingerprinting; behavioural hysteresis; non-disruptive personalized medicine

\section{INTRODUCTION}

We address the possibility to use complexity indices of behavioural variables to identify human subjects. The identification is made by projection of self-similar and normally distributed behavioural data onto Euclidian product spaces based on non-disruptive sensing. This results in a platform that combines data acquisitions provided by body attached sensing devices, mathematical analysis of complexity of the acquired signals, and subsequent projections onto Euclidian spaces representing behavioural states.
Externally applied wearable sensors allow for collecting self-similar time-series of behavioural or physiological parameters such as heart rate, blood oxygenation, skin temperature, steps frequency, and others. The measurements of the behavioural parameters represent surrogate data that characterize patterns through segmentation and a subsequent complexity analysis using data pertaining to expended time spans.

Behavioural surrogate data are segmented and analysed to extract meaningful patterns hidden in the measured time series of behavioural data. The word segmentation is used to indicate a connection between behavioural 
temporal segmentation and image spatial segmentation $[1,2]$. This approach is similar to the concepts of the Gestalt school [1] and psycho-physiologists analysing, e.g., perceptions of images in the retina in the first instants of their arrival $[3,4]$. Segmentation of captured images is aimed at and allows for recognition of well-defined objects from discrete patterns. Similarly, we strive to use complexity indexing to recognize well-defined behaviours that can differentiate various subjects using timediscrete behavioural time-series.

We build geometrical images of behavioural states evolving over time that can be measured and analysed using mathematical tools based on complexity indexing, i.e., scaling properties of self-similar sensory data expressed through the Hurst exponent [5-9].

Our approach shares some fundamental underpinnings with image segmentations, in particular the use of a measure generating dimension [10] and a principle similar to Mumford-Shah functional to verify self-similarity. The Mumford-Shah functional is routinely used in image segmentation as it simultaneously accounts for both high and low dimensions of structural measurements [2].

We first address behavioural indexing, the notion of behavioural indexing and behavioural similarity measures (cf. Section of the Methods).We present application of the proposed approach pertaining to identification of human subjects based on surrogate data using complexity data segmentation including the use of centroids and convex hulls (cf. Section of the Results).

\section{RESULTS}

\section{Human behavioural convex hulls}

The proposed approach to "neuro-fingerprinting" is illustrated using behavioural data of eight healthy subjects selected of a larger pool of healthy humans during their routine daily activities covering roughly up to fifteen hours of sensory data. This time span is divided into roughly two-hours time spans from which the complexity indices are computed and projected onto the behavioural two dimensional product spaces generated by the quantities that are recorded using the VSM sensor. Different colours shown Figure 1 through Figure 6 represent each of the individual's convex hulls encapsulating the surrogate behavioural data. The following numbers are assigned to different colours, i.e., subjects, to make the referencing easier: red/1, green/2, blue/3, violet/ 4 , black/5, grey/6, azure/7, yellow/8.

The results shown at the following visualizations indicate that there is different clustering of subjects with respect to different behavioural product spaces.

The presented results show that each of the subjects posses a different convex hull and centroid as well as different volatility characteristics.

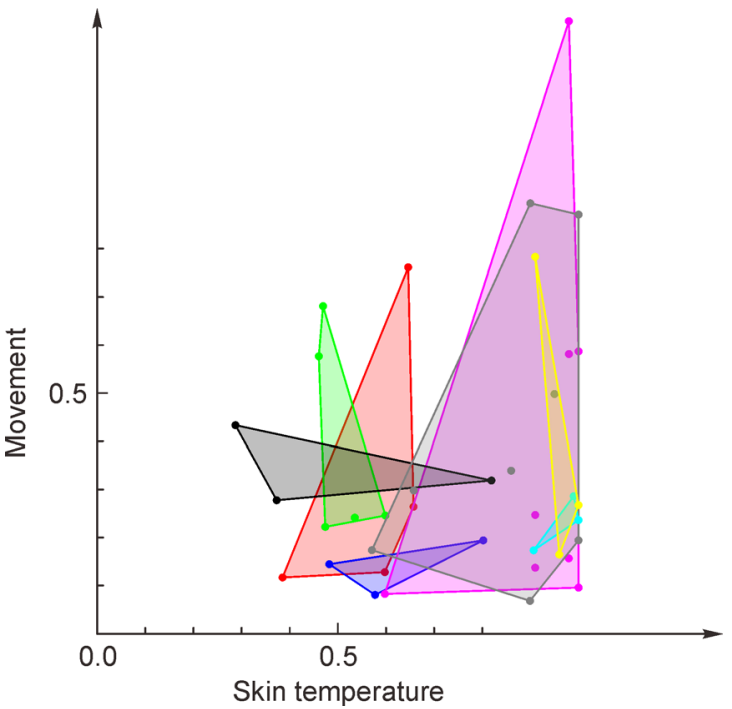

Figure 1. Skin temperature/movement convex hulls of complexity indices. The displayed analysis shows that subjects can be divided, using Table 2, to two similarity clusters. Namely, $\{3,4,6,7,8\}$ and its complement $\{1,2,5\}$. This indicates that, disregarding the centroids and volatility, the two clusters can be viewed as "similar" in terms of the skin temperature and movement complexity indices. The positions of the convex hulls also indicate that a part of the first cluster $\{3,7,8\}$ is positioned in the negative correlation region yielding a higher responsiveness due to the increased irregularities if the generating time-series.

\section{Centroids and volatility indexing}

The four tables included in this section present compounded analysis of human subjects' behavioural similarities or dissimilarities in terms of centroids and volatility of the data to refine the analysis shown at Section of Coarse-grained behavioural patterns in Appendix and to provide a compounded algebraic overview of the subjects' behavioural complexity. The centroid rows correspond to the complexity indices stipulated in the Tables $1-4$ heads.

Consider subjects 4, 5 and 3 to highlight a possible use of the geometric behavioural characterization using the skin temperature/movement data in Table 2. The first subject is a highly trained, the second is a healthy subject and the third is a trained subject. The data for the first subject include running in addition to a daily routine. The data pertaining to the second subject are acquired exclusively during sleep. The data representing the third subject correspond to a non-interrupted high physical activity.

The interpretation of the first subject's data is that it has almost constant complexity of the skin temperature with negative correlation and unbiased complexity of movement. The large volatility (radius) implicates disparity 


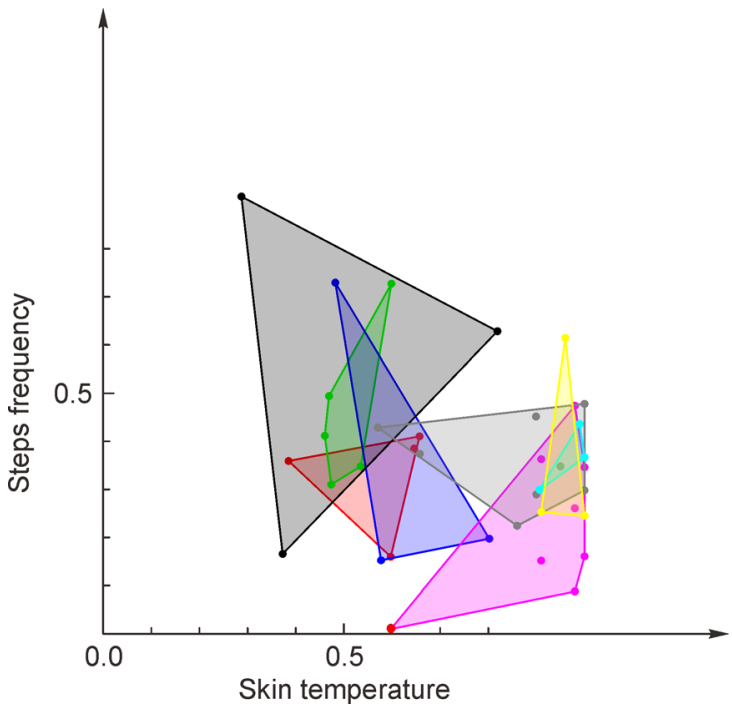

Figure 2. Skin temperature/steps frequency convex hulls. The complexity analysis indicates that, e.g., the subjects 2 and 5 can be viewed as similar according to the behavioural similarity condition (6). The plot also indicates that both of these two subjects made a very small amount of physical movements for their skin temperature and steps frequency exhibit a positive correlation indicating a low complexity of the underlying time-series.

among office work and running behavioural complexity. These numbers may be typical for trained athletes. The second subject's data indicate behavioural conformity with small radius, typical for a rest. The third subject's data show virtually no complexity of the skin temperature and high complexity of movements with almost no volatility. This observation would indicate non-interrupted higher mono-physical activity during a longer period of time. Similar conclusions can be made using the Tables 1-4.

\section{Higher dimensional indexing}

We present an example of a three-dimensional representation of human surrogate data indexing using heart rate; blood oxygenation and steps index space. Figure 7 visualizes an example of such indexing of different human subjects.

Technically, the representation of the surrogate data in higher dimensional spaces is identical to the presentation of the data shown at Section of Coarse-grained behavioural patterns in Appendix.

There is an additional interpretation of the analysis beyond "behavioural fingerprinting". The additional description is associated with the position and volume represented by the convex hulls encapsulating the surrogate data.

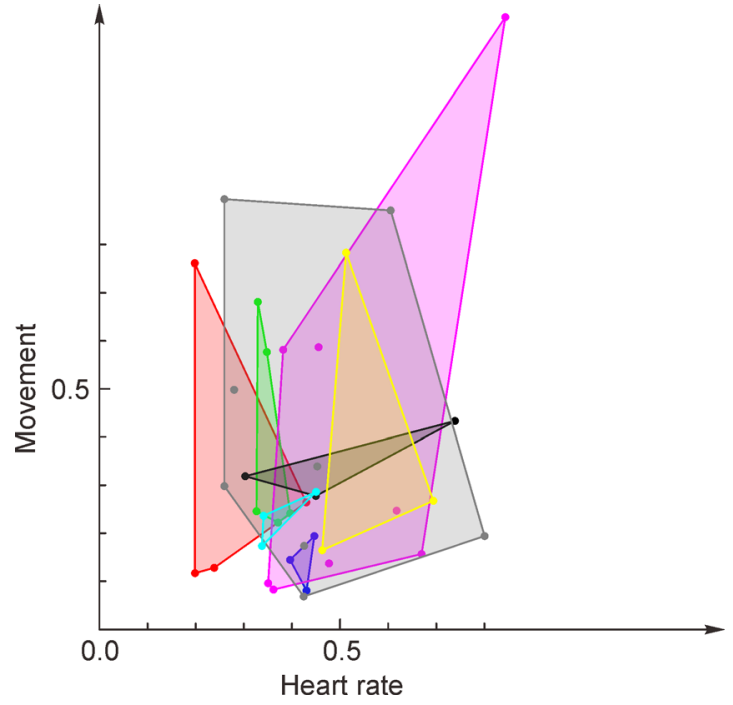

Figure 3. Heart rate/movement convex hulls. The displayed behavioral convex hulls show that, from the point of view of the selected space, there is a very little difference among the subjects using the criterion (6). Since all the convex hulls are positioned inside lower complexity regions we may conclude that the selected group consists of individuals resisting stress in a sense of low movement and low heart rate complexities.

The Brownian point $\left(\frac{1}{2}, \frac{1}{2}, \frac{1}{2}\right)$ gives the centrality in the three-dimensional phase space. Any indices below $\frac{1}{2}$ are pronunciations of negative correlation as mentioned above. This is supposed to indicate more agile responses to stimuli. The indices above the Brownian threshold indicate opposite behavioural patterns. Consequently, we may conclude that it is favourable to observe symmetric and rather low volume convex hulls as a characterization of "healthy" human states.

The example shown in Figure 7 seems to support this conclusion. The green convex hull is that of a highly trained individual while the blue and red ones represent healthy, but less trained, subjects. The subjects were observed during fifteen hours period during regular working day and sleep.

\section{CONCLUSIONS}

The presented results indicate that some of the indices of heart, steps frequency, blood oxygenation, and/or skin perfusion, seem to be unique for each individual. This means that a collection of complexity indices can provide aggregated characterization of factorized groups of patients. This leads to "behavioural fingerprinting". 


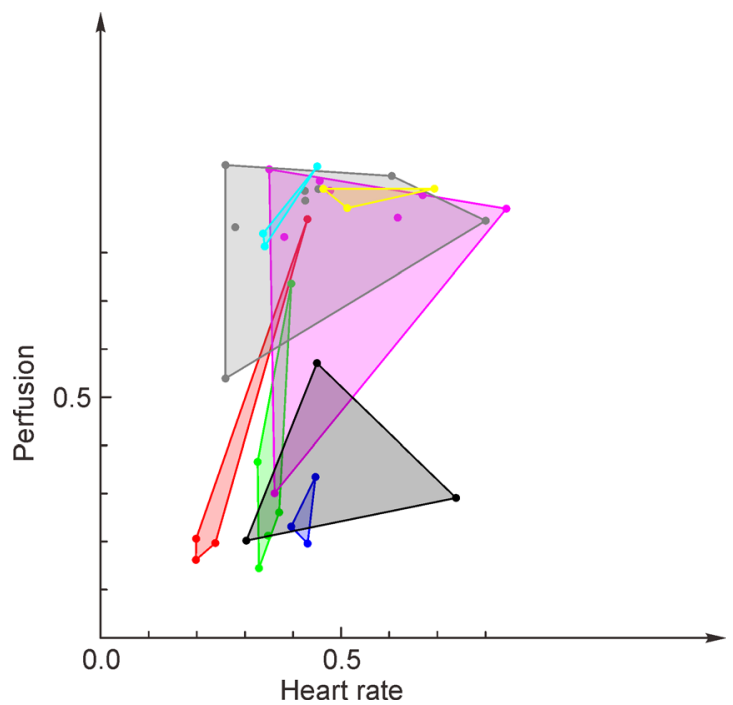

Figure 4. Heart Rate/perfusion convex hulls. The positions of the convex hulls indicate antagonistic tendencies when heart rate and perfusion combination as measured during dally activities. Unlike the analysis shown in Figure 1 this combination clearly indicates subjects' behavioural dissimilarities. Moreover, it also shows that while heart rate complexity tends to be low the complexity of the perfusion data can be higher in stressful environments.

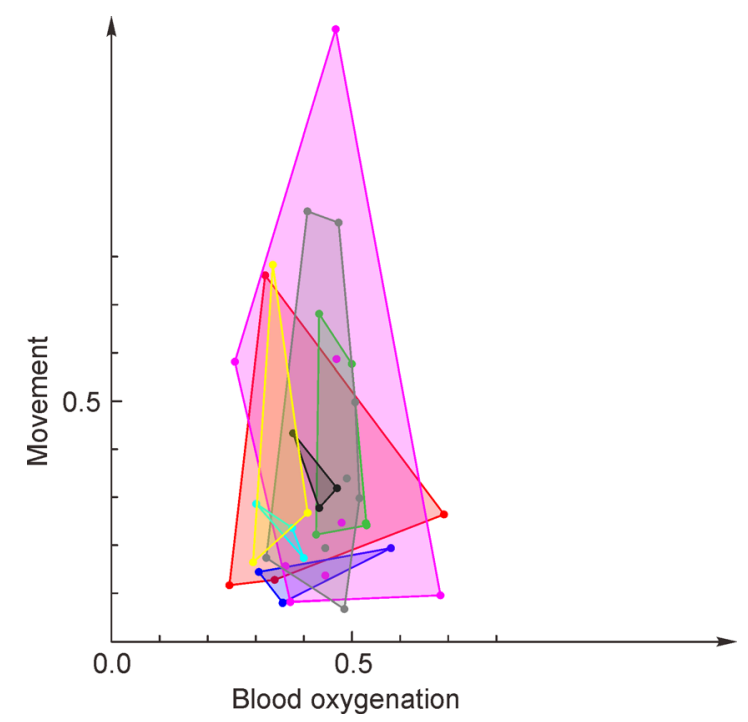

Figure 5. Blood oxygenation/movement convex hulls. The results show, similarly to heart rate/movement index space, that this combination provides less dissimilarity indicators compared to other combinations.

Consequently, the correlation between factorized groups and indexing of their responses to treatment can provide a basis for personalized medicine.

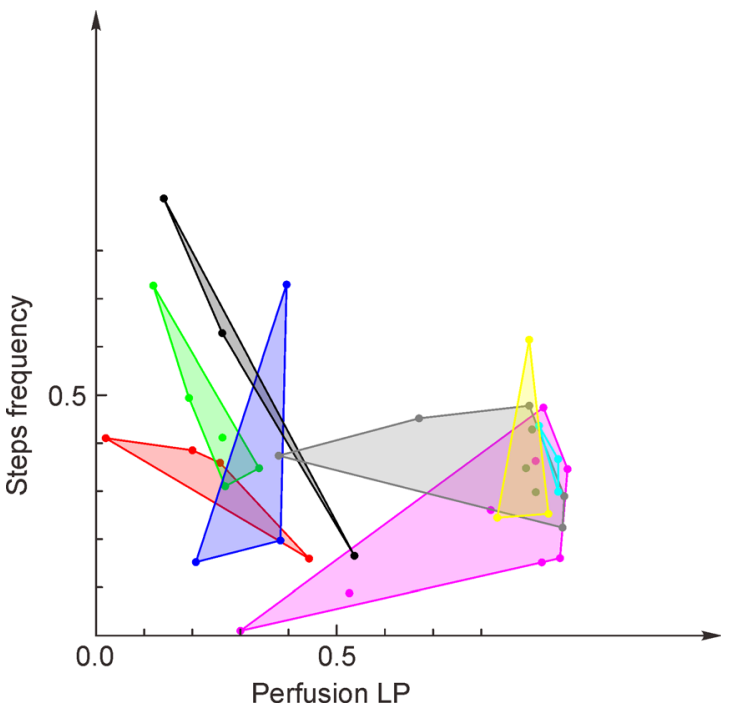

Figure 6. Low pass perfusion/steps frequency convex hulls. The combination exhibits rather high dissimilarities among the subjects. Except for the $\{4,7,8\}$ cluster that other subjects seem to have very little in common from the low passed perfusion and steps frequency combination. The low pass filter is used to analyze the perfusion for the corresponding time series exhibits low frequency and some noise.

\section{METHODS}

\section{Subjects}

The proposed approach is illustrated using behavioural data collected from eight subjects selected from a larger pool of healthy humans. The data were collected at a hospital ward from volunteering medical practitioners.

\section{Behavioural complexity indices}

Behavioural data are obtained using external sensors attached to a subject's body. We use two connected technologies, i.e., (i) micro-electronics based wearable sensors and (ii) mathematics based data processing providing data complexity characterizations. The first item refers to the wearable non-disruptive Vital Signs Monitor (VSM) capable of motion-tolerant monitoring of human behavioural signs without interfering with a person's activities. The second item refers to detection of self-similarity and the underlying scaling properties of some behavioural data using our Cassiopee computational environment.

The index spaces can be viewed as spaces endowed with metric of an ambient Euclidian space. The introduction of the index spaces helps to illustrate the possible deviation of a complexity index of behavioural quantity points from "normal" to a changed functionality of a 
Table 1. Heart rate/movement

\begin{tabular}{lllllllll}
\hline Subject/geometry & $S^{1}$ & $S^{2}$ & $S^{3}$ & $S^{4}$ & $S^{5}$ & $S^{6}$ & $S^{7}$ & $S^{8}$ \\
\hline \multirow{2}{*}{ Centroid } & 0.275866, & 0.343896, & 0.424401, & 0.542258, & 0.497176, & 0.515668, & 0.376352, & 0.556469, \\
\multirow{2}{*}{ Radius } & 0.37617 & 0.405222 & 0.13964 & 0.430708 & 0.343213 & 0.425382 & 0.23194 & 0.40508 \\
\hline
\end{tabular}

Table 2. Skin temperature/movement

\begin{tabular}{lllllllll}
\hline Subject/geometry & $S^{1}$ & $S^{2}$ & $S^{3}$ & $S^{4}$ & $S^{5}$ & $S^{6}$ & $S^{7}$ & $S^{8}$ \\
\hline \multirow{2}{*}{ Centroid } & 0.559974, & 0.510095, & 0.620452, & 0.836843, & 0.493034, & 0.846449, & 0.965417, & 0.956527, \\
\multirow{2}{*}{ Radius } & 0.344589 & 0.393477 & 0.13964 & 0.486973 & 0.343213 & 0.428527 & 0.23194 & 0.40508 \\
\hline
\end{tabular}

Table 3. Skin temperature/steps frequency

\begin{tabular}{lllllllll}
\hline Subject/geometry & $S^{1}$ & $S^{2}$ & $S^{3}$ & $S^{4}$ & $S^{5}$ & $S^{6}$ & $S^{7}$ & $S^{8}$ \\
\hline \multirow{2}{*}{ Centroid } & 0.28927, & 0.398698, & 0.13964, & 0.449629, & 0.343213, & 0.46879, & 0.23194, & 0.40508, \\
\multirow{2}{*}{ Radius } & 0.339668 & 0.502392 & 0.359726 & 0.153689 & 0.56756 & 0.368654 & 0.367277 & 0.370743 \\
\hline
\end{tabular}

Table 4. Blood oxygenation/steps frequency

\begin{tabular}{lllllllll}
\hline Subject/geometry & $S^{1}$ & $S^{2}$ & $S^{3}$ & $S^{4}$ & $S^{5}$ & $S^{6}$ & $S^{7}$ & $S^{8}$ \\
\hline \multirow{2}{*}{ Centroid } & 0.418802, & 0.475524, & 0.414286, & 0.473795, & 0.426038, & 0.443137, & 0.3587, & 0.345815, \\
Radius & 0.37417 & 0.383454 & 0.13964 & 0.429305 & 0.343213 & 0.348983 & 0.23194 & 0.40508 \\
\hline & 0.399662 & 0.300909 & 0.175116 & 0.843758 & 0.102236 & 0.546713 & 0.0792642 & 0.378251 \\
\hline
\end{tabular}

particular subsystem [11]. Hence, the Euclidian distance from whatever we considered "normal" may indicate presence, severity, or progression of behavioural deviation resulting in behavioural diagnostics of symptoms and signs both in the context of normality and disease.

Self-similar normally distributed surrogate data are analysed by constructing a map from the space of behaviours onto the space of complexity indices that correspond to an approximate non-integer HausdorffBesicovitch dimensions of finite time series of a subject's behavioural patterns.

The mathematical foundations of the proposed indexing framework rely on two fundamental assumptions. The first assumption is that stochastic normally distributed self-similar processes characterise at least some behavioural patterns. Thus, each time-series must be analysed to verify its self-similarity and normal distribution. Consequently, if these properties are confirmed we apply various ways to find the non-integer HausdorffBesicovitch dimensions of these patterns and the corresponding scaling laws. From the point of view of clinical implementations, the unavoidable limitation of the finiteness of the sensory readings must be taken into account.

The second assumption is that of ergodicity. It is required to compare surrogate data obtained from many different individuals both with respect to individual time averaging procedures as well as subjects sampling to obtain the power scaling laws.

\section{Surrogate data segmentation}

It is important to identify time and/or space points at which self-similarity is changing, i.e., when it is lost or its scaling properties change. These behavioural discontinuities indicate behavioural pattern changes that may reflect changes of feedback control of the central nervous system in response to internal and/or to external stimuli. The result presented in Figure 8 is based on the heart rate that appears to have required self-similarity and normal distribution.

\section{Behavioural index spaces}

An example of an index is temperature. Increasing temperature indicates corrective physiological actions. Increasing value of an index indicates transition from anomalous to super diffusion. Lower values of an index indicate just the opposite that we relate to altered behavioural patterns. This is how we can infer from macroscopic observables the nature of the microscopic structure of stochastic processes associated with certain 


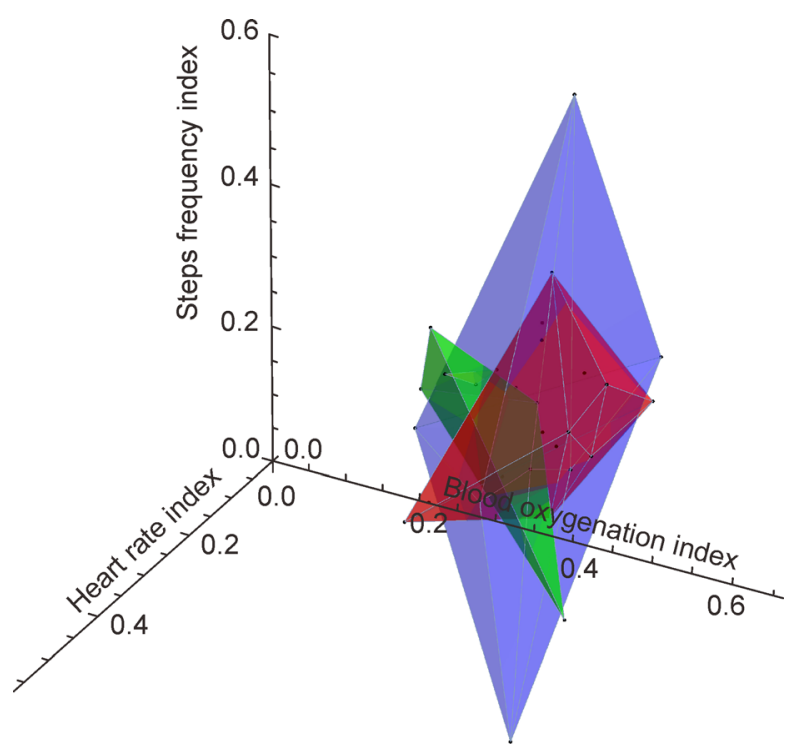

Figure 7. Convex hulls of computed indices in Euclidian Space using human surrogate data based on fifteen hours of sensory data collection. The space is based on heart rate, oxygenation and steps frequency.

physiological states.

Consider the following example shown in Figure 9 that is based on sensory data of a healthy subject during typical professional activities during a working day. We partition the heart rate indices/blood oxygenation indexes space that is contained in $(0,1)^{2}$ into four quadrants.

The quadrant partitioning is cantered at what we call the Brownian point. The arguments behind this approach are that the Brownian point corresponds to standard Brownian functions given by Wiener processes. The lower left quadrant corresponds to $H<1 / 2$ on both axes that represent negatively correlated surrogate data, i.e., data with lots of irregularity. We interpret this quadrant clinically as anxiety/high activity region. The upper right quadrant $(1 / 2,1) \times(1 / 2,1)$ represents Hurst indices yielding positive correlations, i.e., more data regularity. We interpret this quadrant as the relaxed region. The remaining two quadrants contain indices representing mixture of positively and negatively correlated processes. Since the central nervous system can balance such inputs we denote these regions as neutral regions.

The indexing shown in Figure 9 holds the key to the presented concept. We construct convex hull of the computed complexity indices of the time-segmented data including its centroid and radius to obtain dissimilarity measures providing behavioural identification of human subjects.

\section{Behavioural identification}

We construct behavioural complexity convex hulls as the main tool to identify human subjects using behavioural time series. The behavioural complexity convex hulls combined with dissimilarity indicatrices seem to be strong

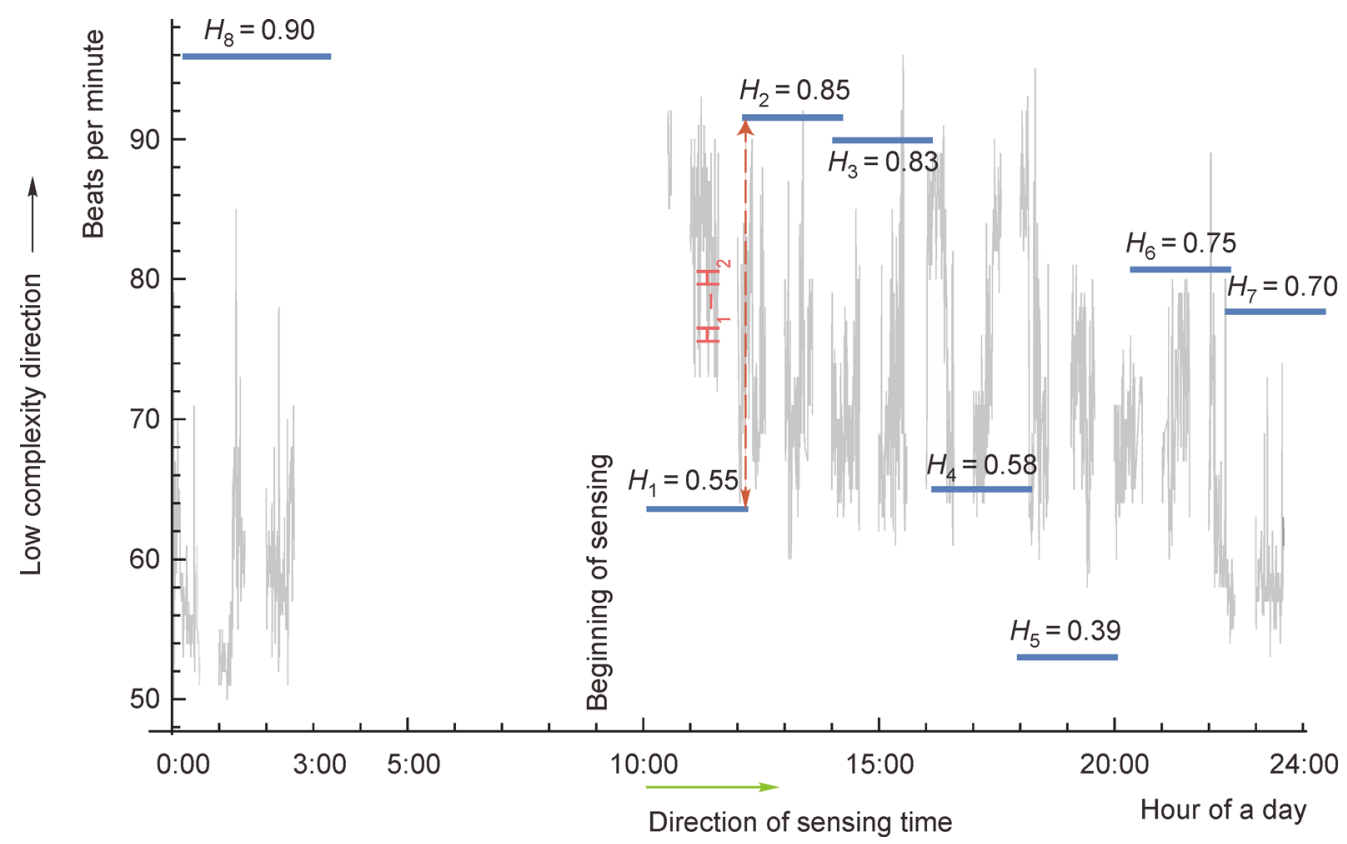

Figure 8. Heart rate surrogate data segmentation. The step function, in blue, correlates only to the time axes. It has no relation to the $y$-axis. It represents the values of the Hurst exponents computed from 123 data points corresponding to about 15 hour based stroboscopic frequency of 30 seconds. Some data are removed due to either missing signal from the sensor or an extensive noise detected in the surrogate data. 


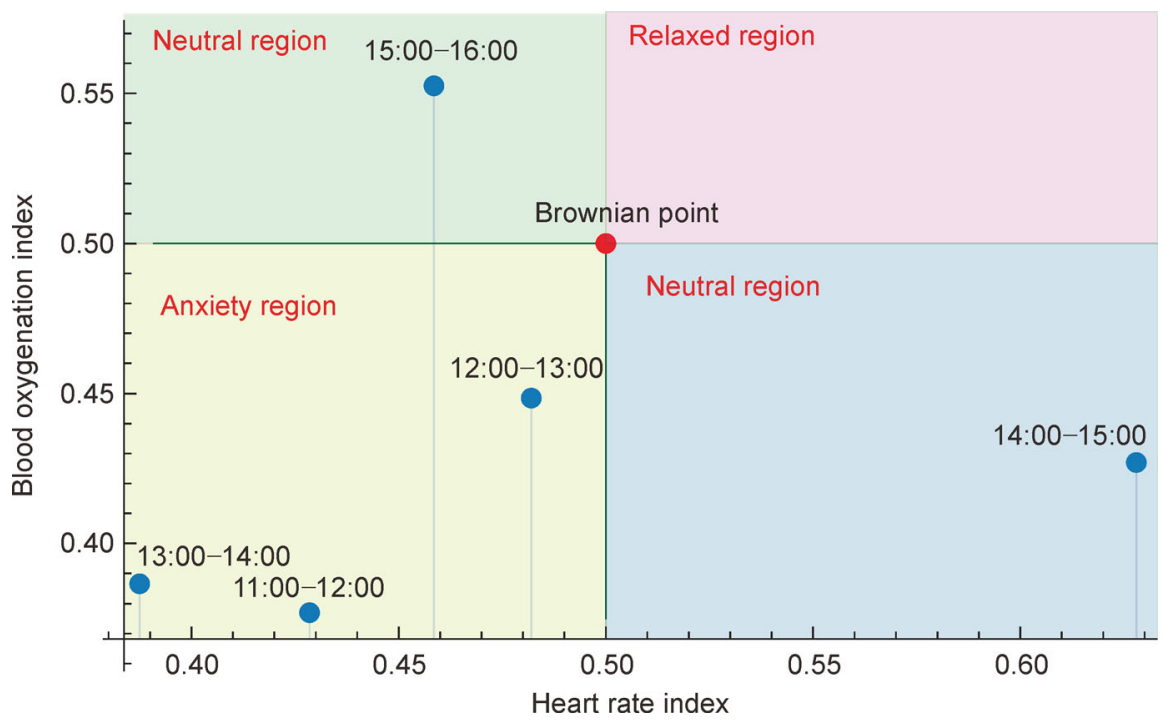

Figure 9. Heart rate/Blood oxygenation index space. The plot shows Euclidian space and its partitioning into anxiety, relaxed and neutral regions with the Brownian point at the center of the unit square. The Brownian point, corresponding to the point (1/2, 1/2), represents processes that can be modeled by standard Brownian functions that do not exhibit negative nor positive correlations. The presented representation corresponds to aggregated measures of surrogate data based on the analysis of self-similarity, and, consequently using scaling properties that can be characterized by fractional dimensions. The presented results are based on surrogate human data during routine daily activities.

enough to distinguish among human subjects.

The application of complexity measures to indexing behavioural patterns on which the presented approach to neural-fingerprinting is based follows upon [12] and in some of the citations therein.

Consider $1<m \in \mathbb{N}$ human subjects. Assume we compute $1<n \in \mathbb{N}$ complexity indices either by surrogate data segmentation or by repeated measurements. We denote by $H^{j} \subset(0,1)$ a computed set of complexity indices generated by surrogate data corresponding to the $j$-th subject.

Thus, let

$$
\mathcal{H}^{j}=\left\{h_{i}^{j} \mid i=1, \ldots, n\right\}, j=1, \ldots, m,
$$

and, let ${ }^{1)}$

$$
\mathcal{H}_{\text {convex }}^{j}=\text { Convex } \operatorname{Hull}\left\{\mathcal{H}^{j}\right\}
$$

\section{Behavioural similarity indicatrix}

Let us consider a pairwise similarity indicatrix given by

$$
\delta_{k, j} \equiv \frac{\operatorname{meas}\left(\mathcal{H}_{\text {convex }}^{j} \cap H_{\text {convex }}^{k}\right)}{\operatorname{meas}\left(\mathcal{H}_{\text {convex }}^{k}\right)} .
$$

It follows from (3) that if $\mathcal{H}_{\text {convex }}^{k} \cap H_{\text {convex }}^{j}=\varnothing$ then $\delta_{k, j}$ $=0$. If $\mathcal{H}_{\text {convex }}^{k} \subseteq H_{\text {convex }}^{j}$ then $\delta_{k, j}=1$. Let us consider the opposite case when

$$
\mathcal{H}_{\text {convex }}^{j} \subseteq H_{\text {convex }}^{k} .
$$

Then

$$
\delta_{k, j} \leqslant 1, \delta_{j, k}=1
$$

The non-trivial intersection, i.e., when

$$
\mathcal{H}_{\text {convex }}^{k} \cap H_{\text {convex }}^{j} \neq \varnothing,
$$

yields $\delta_{k, j} \leqslant 1$.

\section{Behavioural geometric similarity}

In addition to the similarity indicatrices we also compute the centroid and radius of the super-scribed circles to a given convex hull of behavioural complexity indices. We assign a centroid to each $H_{\text {Convex }}^{j}$ by computing

$$
\frac{\int s \partial \mathcal{H}_{\text {Convex }}^{k}(s) d s}{\int \partial \mathcal{H}_{\text {convex }}^{j}(s) d s} \text {. }
$$

Simultaneously, we can determine the radius of the smallest disk covering $\mathcal{H}_{\text {Convex }}^{j}$ with the centre at the centroid. While the centroid represents position of the convex hull in a selected Euclidian space representing

\footnotetext{
1) The convex hull is the smallest convex manifold encapsulating a given set of computed indices.
} 
Table 5. Summary of different combinations of behavioral indicatrices, centroids and areas of behavioural convex hulls contributing to evaluation of subjects' similarity likelihoods.

\begin{tabular}{llll}
\hline Indicatrix & Centroids & Areas & Similarity likelihood \\
\hline 0 & Any & Any & None \\
1 & Inside the enclosing convex hull & Imbedded & Positive \\
$\leqslant 1$ & Inside the enclosing convex hull & Satisfy the convergence criterion (7) & Very high \\
\hline
\end{tabular}

behavioural indices, the radius represents the volatility of the data. The centrality and the data spread, i.e., volatility, are meant to provide a more detailed characterization of a nontrivial subsection of convex hulls.

Assume Equation (4) holds. We propose that two subjects can be "likely" behaviourally similar if

$$
\operatorname{Centroid}\left(\mathcal{H}_{\text {convex }}^{k}\right) \in \mathcal{H}_{\text {convex }}^{j}
$$

and

$$
\operatorname{meas}\left(\mathcal{H}_{\text {convex }}^{k}(n)\right) \rightarrow \operatorname{meas}\left(\mathcal{H}_{\text {convex }}^{j}(n)\right) \text { as } n \uparrow \text {. }
$$

The argument $n$ corresponds to a number of data points.

The requirement Equation (7) is related to the consistency of the temporal behavioural partial overlap of two subjects. The consistency requires that data contained in a non-trivial intersection given by Equation (4) must be stable in the sense

$$
\operatorname{meas}\left(\mathcal{H}_{\text {convex }}^{j} \cap \mathcal{H}_{\text {convex }}^{k}\right)>0
$$

as $\operatorname{Card} \mathcal{H}_{\text {convex }}^{j}, \operatorname{Card} \mathcal{H}_{\text {convex }}^{j}$ increase with the number of the behavioural data points with respect to either increased time span of measurements of repeated sensing. Condition Equation (8) requires that a certain amount of data be shared among different subjects.

The combination of the values of behavioural indicatrices, centroids, and areas of behavioural convex hulls, i.e., data volatility, yielding similarity predictions is summarised by Table 5 .

\section{AUTHORS' CONTRIBUTIONS}

PK developed the mathematical framework as well as the computational eco-system, Cassiopee, to compute and to analyse the behavioural complexity indices yielding the possibility of "behavioural fingerprinting". AvG provided initial impulse to use our previous work [5] to address the possibility of the behavioural fingerprinting. He also provided the clinical expertise to test our approach.

\section{ACKNOWLEDGEMENTS}

The authors were supported in part by Biovotion, AG, that provided the sensors and access to the raw sensory data. Our activities were supported in part by the Service de Psychiatrie de l'Age Avance that is part of Centre hospitalier universitaire Vaudois and by University of Lausanne, Switzerland.

\section{COMPLIANCE WITH ETHICS GUIDELINES}

The authors Petr Kloucek and Armin von Gunten declare that they have no competing interests.

The authors declare that

(i) The study was carried out in accordance with the Declaration of Helsinki and CIOM;

(ii) All subjects gave their written informed consent

The authors declare that the presented research includes observational research only with no video or participation by the authors of this study. Consequently, this communication is exempt from an Institutional review board review. They refer to Code of Federal Regulations of the United States of America, Part 46, Protection of Human Subjects, and Revised on January 15,2009 .

\section{APPENDIX}

\section{Coarse-grained behavioural patterns}

Our approach is based on the assumption that indexing of behavioral patterns provides objective and quantitative measurements of humans. The concept itself stems from and relies on not only on mathematical frameworks yielding macroscopic characterizations of physical systems based on their microscopic properties but on surrogate pronunciations of brain activities. This type of coarse-graining approach has been used many times in fluids or smart materials with remarkable success throughout the past century based on averaging procedures. We replace these averaging techniques by coarsegraining provided by physiological and behavioral macroscopic data that can be gathered by external biosensors.

In our approach, the microscopic level is represented by tiny changes in physiological readings that we map onto the Hausdorff-Besicovitch dimension. This number can be thought of as a macroscopic system parameter. The indices we introduce to objectively measure various physiological effects and/or neuropathology can be thought of as being subsumable and describable through one single measure: similar to the entropy or temperature.

Consider temperature. Temperature is a macroscopic statistical quantifier and so are the indices we consider. Temperature itself cannot indicate a specific disease or etiology. Nevertheless, it is routinely used as a forefront diagnostic tool to judge physiological states. The indices we introduce, such as heart rate, perfusion or blood oxygenation, are stochastic processes that can be thought of as a generalization of the temperature analogy to gauge physiological states.

Longitudinal measures are the only way to observe 


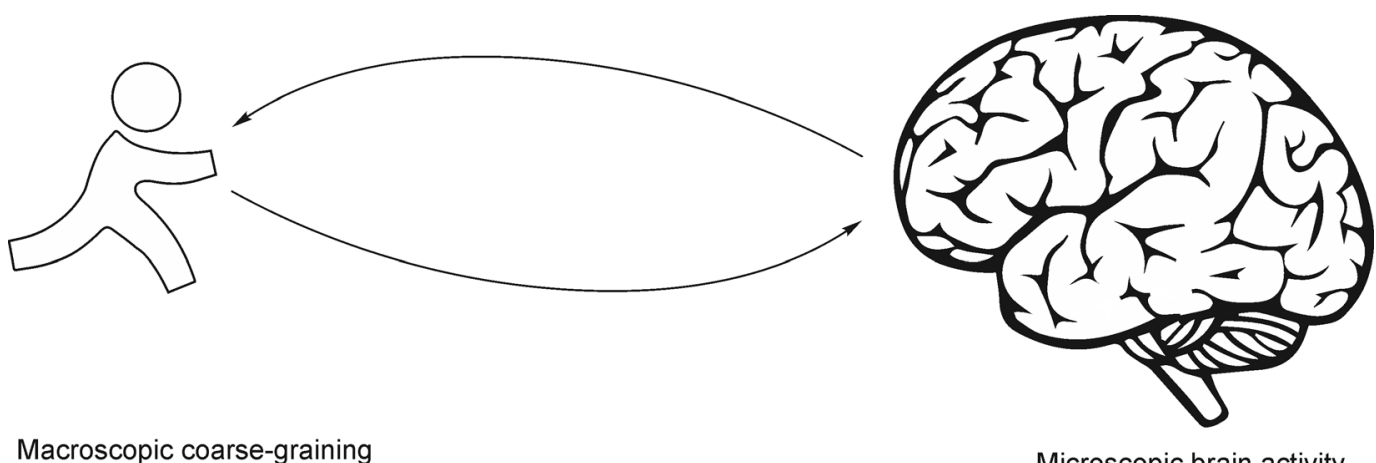

surrogate data analysis

Microscopic brain activity

Figure A1. Behavioral coarse-graining of autonomous nervous system. We consider the brain as a social organ providing microscopic patterns that might prove difficult to analyze similar to tracking every molecule in a fluid. The behavioral pronunciations of its directives in terms of surrogate, macroscopic, data lend its self to manageable mathematical analysis through complexity longitudinal measures.

transitions and to decide if a given physical/physiological/ behavioral/psychopathological variable possesses a selfsimilar organization expressed by a dimension generating measure. These techniques provide objective measures of complexity of behavioral patterns similar to those that can be generated synthetically, e.g., using fractional Brownian motion. We observe that a loss or a change of behavioral self-similarity is an essential part of unique human behaviors.

\section{Behavioural Indexing}

Considered a discrete one-dimensional fractional Brownian motion composed of a large number of coordinates generated by the Hurst index $1 / 2$. The non-integer Hausdorff-Besicovitch dimension, $D$, of its graph in two dimensional $x-y$ Euclidian coordinate system would be close to $3 / 2$ [13]. The fractional dimension $D$ and the Hurst index are related by $D=d-H$ where $d$ is the dimension of the ambient space, assuming time-equidistant discrete fractional Brownian process.

As $H \rightarrow 0$ we obtain more and more irregular structures. In the other case $H \rightarrow 1$ just the opposite is true. The interpretation of the meaning of different indices of acquired surrogate macroscopic sensory data is as follows. If the Hurst index is smaller than $1 / 2$ then the increments of considered processes are negatively correlated [10] and positively correlated in the other case. Positive correlation implies higher probability of correct estimates of some future states and vice versa. In our settings the positive correlation implies that when $H$ $>1 / 2$ we observe diminishing variance of surrogate behavioural patterns ${ }^{1)}$.
We interpret surrogate self-similar normally distributed sensory data with diminishing or increasing variance as an indication of different behavioural states that seems to be unique to a human subject.

\section{REFERENCES}

1. Koenderink, J. J. (1984) The structure of images. Biol. Cybern., 50, 363-370

2. Morel, J. M. and Solimini, S. (1995) Variational Methods in Image Segmentation. USA: Birkhauser Boston Inc

3. Julesz, B. (1980) Spatial nonlinearities in the instantaneous perception of textures with identical power spactra. Philos. Trans. R. Soc. Lond., 290, 83-94

4. Julesz, B. (1981) Textons, the elements of texture perception, and their interactions. Nature, 290, 91-97

5. Peitgen, H.-O., Jrgens, H. and Saupe, D. (1992) Chaos and Fractals. New York: Springer-Verlag

6. Ness, M. V. (1968) Fractional Brownian motions, fractional noise and application. SIAM Rev., 10, 422-437

7. Mandelbrot, B. B. (1997) Fractals, Form, Chance and Dimension. San Francisco: W. H. Freeman and Company

8. Bassingthwaighte, J. B., Liebovitch, L. S. and West, B. J. (1994) Fractal Physiology. New York: Oxford University Press

9. West, B. J. (2010) Fractal physiology and the fractional calculus: a perspective. Front. Physiol., 1, 12

10. Preiss, D. (1987) Geometry of measures in Rn: distribution, rectifiliability and densities. Ann. Math., 125, 537-643

11. Hassabis, D. and E. A. Maguire, (2009) The construction system of the brain. Phil. Trans. R. Soc. B. Biol. Sci., 364, 1263-1271

12. Kloucek, P., P. Zakharov, and A.von Gunten, Indexing of Behavioural Complexity Using Self-similar Surrogate Data. Preprint, 2016

13. Morters, P. and Peres, Y. (2010) Brownian Motion. Cambridge Series in Statistical and Probabilistic Mathematics. Cambridge: Cambridge University Press

\footnotetext{
1) Note that correlation does not imply causality.
} 